\title{
Air-structured optical fiber drawn from a 3D- printed preform
}

Article in Optics Letters · September 2015

DOI: $10.1364 /$ OL.40.003966

CITATIONS

6

8 authors, including:

\section{John Canning}

University of Sydney

519 PUBLICATIONS $\quad 4,607$ CITATIONS

SEE PROFILE

Yanhua Luo

UNSW Sydney

118 PUBLICATIONS 915 CITATIONS

SEE PROFILE
READS

294

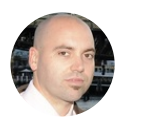

Sergio G. Leon-Saval

University of Sydney

172 PUBLICATIONS 2,764 CITATIONS

SEE PROFILE

Gang-Ding Peng

UNSW Sydney

494 PUBLICATIONS $\quad 4,159$ CITATIONS

SEE PROFILE

Some of the authors of this publication are also working on these related projects:

Project $\quad$ Specialty polymer and silica optical fibre View project 


\title{
Air-structured optical fibre drawn from a 3D-printed preform
}

\author{
Kevin Cook $^{1}$, John Canning ${ }^{1 *}$, Sergio Leon-Saval ${ }^{2}$, Zane Reid ${ }^{1,+}$, Md Arafat \\ Hossain ${ }^{1}$, JADE-Edouard ComatTI ${ }^{1}$, YANHUA Luo ${ }^{3}$ AND GANG-DING Peng ${ }^{3}$ \\ ${ }^{1}$ interdisciplinary Photonics Laboratories (iPL), School of Chemistry, The University of Sydney, NSW 2006, Australia; \\ ${ }^{2}$ School of Physics, The University of Sydney, NSW 2006, Australia; \\ ${ }^{3}$ National Fibre Facility, School of Electrical Engineering and Telecommunications, UNSW, Sydney, NSW 2052, Australia; \\ ${ }^{+}$Currently with Precision 3D Printing (P3P), Unit 2, 28 Anvil Road, Silverdale, Auckland, New Zealand. \\ *Corresponding author: john.canning@sydney.edu.au
}

Received XX Month XXXX; revised XX Month, XXXX; accepted XX Month XXXX; posted XX Month XXXX (Doc. ID XXXXX); published XX Month XXXX

\begin{abstract}
A structured optical fibre is drawn from a 3D-printed structured preform. Preforms containing a single ring of holes around the core are fabricated using filament made from a modified butadiene polymer. More broadly, 3D printers capable of processing soft glasses, silica and other materials are likely to come on line in the not-sodistant future. 3D printing of optical preforms signals a new milestone in optical fibre manufacture. (C) 2015 Optical Society of America
\end{abstract}

OCIS codes: (060.2280) Fiber design and fabrication; (060.4005) Microstructured fiber; (130.5460) Polymer waveguides; (050.6875) Three-dimensional fabrication.

http://dx.doi.org/10.1364/OL.99.099999

In recent years, there has been an explosion of interest in 3D printing technologies and there is now a vast range of 3D printing methods that are finding new applications every day in a whole host of areas from science and engineering to medicine and the arts [1]. They are set to revolutionise manufacturing. Fused deposition modelling (FDM) is one of the most commonly used techniques, being the first and simplest demonstration of 3D printing in the late 1980s [2]. It is especially suited for instrument prototype casings such as those required for novel smartphone spectrometers [3]. In FDM, a polymer is fed through a heated nozzle which melts the polymer - essentially a fancy thermal glue gun using a finite extrusion nozzle with programmable $x y z$ positioning capability. Optical polymerisation methods using both light emitting diodes (LEDs) and lasers offer higher resolution and are increasingly popular; however, they do not have the same material range given the specific absorption and monomer polymerization requirements. Selective laser sintering (SLS) is another popular technique where a high power laser is utilised to fuse not only plastic, but glass, metal or ceramic particles or powders into 3D objects [4]. A 3D profile is achieved by scanning the laser on the horizontal plane layer-by-layer, lowering the printing bed between each layer. All these methods have their particular rate limiting steps determined by the consolidation process details and the need for full $x y z$ scanning. More recently, the technique of continuous liquid interface production (CLIP) promises significantly accelerated production of the 3D printed object by removing the requirement for $x y$ scanning [5]. In this approach, light is projected into a reservoir of UV curable resin and a build platform moves continuously in the vertical direction to grow an object. FDM, SLS and other related variants are experiencing tremendous growth world-wide, particularly for rapid prototyping and manufacturing. 3D printing is also being applied to cellular assembly processing of biomedical interest [6]. 3D printing technologies are reaching most fields, including more recently photonics. For example, a company called Luxexcel is already producing high transparency Fresnel lenses by printing with poly(methyl methacrylate) (PMMA) filament [7]. Most interesting, is recent work demonstrating directprint short, solid plastic fibre [8] and "light pipes" [9] using transparent polymers, the first 3D printed waveguides.

Here, we explore harnessing 3D printing for optical fibre fabrication. We see it as revolutionising the manufacture of all optical fibre fabrication, including glass optical fibres as 3D glass printing comes on line $[10,11]$. Whilst there is debate as to the merits of the technology for conventional step-index optical fibres and how dopant distributions might be 3D printed, one breed of optical fibre that could particularly stand to benefit immediately are so-called structured (both microstructured and nanostructured) optical fibres. The fabrication of these fibres is limited to methods that are often suitable for specific materials. The stack-and-draw technique, where capillaries are manually assembled into a hexagonal structure prior to drawing [12], is the most popular method but is obviously limited to hexagonally packed periodic structures, giving rise to so-called photonic crystal fibres. More complex structures such as Fresnel $[13,14]$ and related fractal fibres [15] cannot be easily constructed by assembling capillaries and have required other methods including drilling [12], extrusion [16,17] and injection molding [17]. As can be imagined, these methods are currently all time-consuming and 3D printing offers an immediately competitive advantage, and certainly for polymer fibres where 3D printing manufacturing is most mature.

In this paper, an alternative approach to making structured fibres is explored by utilising a 3D printer to design and print a structured preform that is then drawn to fibre. As proof of principle, the more mature FDM printing method is chosen to print a preform using a transparent thermosetting polymer that is subsequently drawn to fibre. An arbitrary fibre geometry consisting of a solid core surrounded by 6 air holes is chosen to assess the 3D-printed preform approach. The material used is a commercially available 3D printing filament consisting of a propriety polystyrene mixture containing styrenebutadiene-copolymer and polystyrene, labelled here as SBP. SBP can offer several significant advantages when compared to other polymer filaments: it is transparent, it has a high degree of flexibility and it does not suffer from discoloration under stress or when bent. A low-cost, commercially-available thermosetting 3D printer is used to print the fibre preform with this filament. The preform can be subsequently drawn to fibre with relative ease, without the need for pre-annealing or hole-pressurisation, maintaining its cross-sectional features after being drawn to fibre form. We also show that preform transparency can be improved by annealing which removes the scattering component arising from the printed interfacial layers and bubbles trapped during printing.

The aim of this paper is to demonstrate the first 3D printed structured optical fibres, choosing to focus on existing polymer based 3D printing as proof of concept. As new materials such as soft glasses 
and silica glasses become accessible to 3D printing, all fibre technologies will eventually be encompassed. In the meantime, structured polymer fibres have significant potential in their own right within a range of application areas. For example, chirped graded Fresnel fibre designs could find applications in LANs where step-index fibres are traditionally used [18]. Microstructured polymer optical fibre also shows strong potential in biomedical applications due to polymer compatibility with organic materials. Basic structured fibres with only a small number of holes offers cheap, biocompatible attenuation sensors for applications such as orthodontic pain monitoring within the human organism [19].

The air-structured preform was designed on Inventor computer aided design (CAD) software. Fig. 1 shows a depiction of the preform shape and a photo of the 3D printed preform end. The length of the preform was chosen to be $L=10 \mathrm{~cm}$, although given the volume workspace of the 3D printer used (a Deltasine "Redback-2"), lengths of up to $L=39.5 \mathrm{~cm}$ can be achieved. The preform diameter of $\phi=1.6 \mathrm{~cm}$ was chosen in order to be compatible with the polymer fibre draw tower and furnace. The solid core of the preform is surrounded by 6 air holes each with diameter $\phi$ hole $=0.2 \mathrm{~cm}$ and evenly separated along the periphery. A low-cost, commercially-available 3D thermal printer was used to print the preform. The Redback- 2 is a delta design model which is ideally-suited for printing circular structures compared to traditional $x y z$ printers. The SBP polymer filament has a higher degree of transparency (specified $>90 \%$, loss $\alpha<1.2 \mathrm{~dB}$ ) when compared to the more commonly used acrylonitrile butadiene styrene (ABS) polymer. The extruder and bed temperatures of the 3D printer were set at $T_{e x}=210^{\circ} \mathrm{C}$ and $T_{\text {bed }}=95^{\circ} \mathrm{C}$ respectively. The preform used in the following work was printed laterally with the preform on its side; however, it is also possible to print vertically. Resolution of the printing on the $x y$-axes was set to $\Delta x=\Delta y=0.4 \mathrm{~mm}$ and $\Delta z=0.1 \mathrm{~mm}$ on the $z$ axis. For the case reported here the printing time was $t=6$ hours (all automated, so no further steps need to be taken by the fabricator).
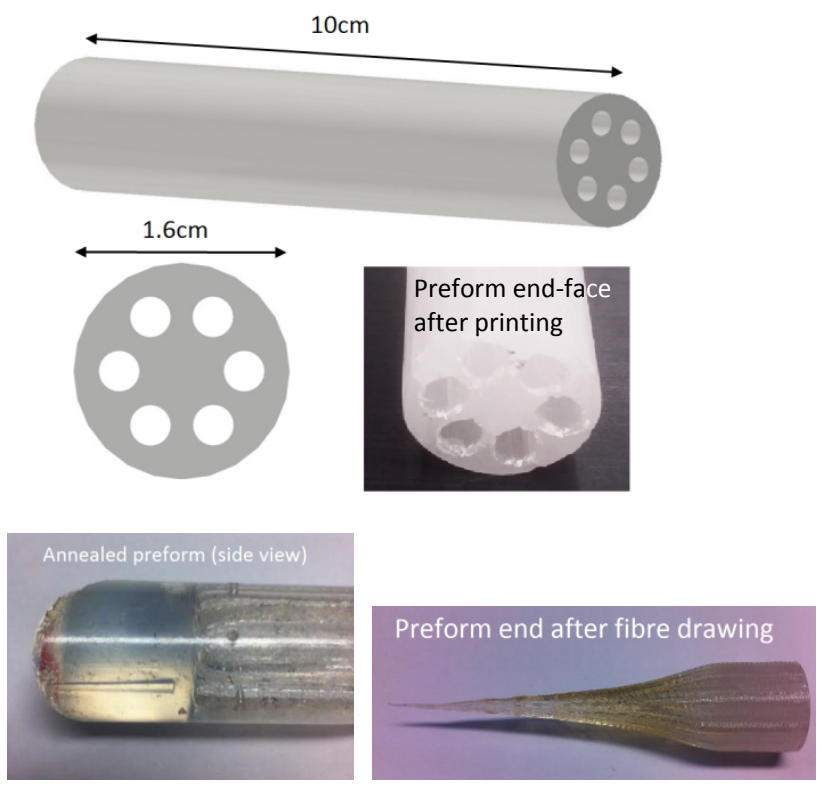

Fig. 1. Illustration of SBP fibre preform design and photos of annealed preform and preform after fibre draw.

Much of the observed opaqueness of the printed preform arises from scattering at the interface layer edges created by printing. However, it was found that drawing into optical fibre removed much of this and improved transparency such that post-annealing is not necessary. Annealing itself can remove significant scattering and dramatically improve transparency through the preform, making it easier to quantify attenuation and absorption spectroscopically. To demonstrate this, a second preform was printed, the only difference in design being that each end of the preform had a $2 \mathrm{~cm}$ long solid polymer end-cap in order help the preform maintain its circular shape during annealing. The preform was held in a computercontrolled oven under a vacuum $\left(P=2.3 \mathrm{kPa}, T=128{ }^{\circ} \mathrm{C}, t=96 \mathrm{hrs}\right)$. This was followed immediately by a secondary annealing step $(T=$ $\left.148{ }^{\circ} \mathrm{C}, t=48 \mathrm{hrs}\right)$ and a third $\left(T=168{ }^{\circ} \mathrm{C}, t=96 \mathrm{hrs}\right)$. The reason for this three step process is that the exact annealing conditions for SBP polymer were unknown; therefore various temperatures had to be tested. A starting temperature of $128{ }^{\circ} \mathrm{C}$ was chosen since this was slightly higher than the glass transition temperature of $A B S, T_{g}=$ $(105-115){ }^{0} \mathrm{C}$ [20], and SBP is known to be a modified version of ABS. The preform was visually inspected for transparency after each annealing step, with two increases of $20{ }^{\circ} \mathrm{C}$ required before the preform was found to be visibly transparent as shown in figure 1. Further optimization of these annealing conditions will be the subject of future work.

After annealing, a $4 \mathrm{~mm}$-thick slice was cut from the preform and polished using optical grade polishing paper. The transmission spectra were recorded in a spectrophotometer $(\lambda=200$ to $900 \mathrm{~nm})$. Another identical sample was taken from a third, non-annealed preform and similarly polished for comparison. The transmission spectra in Fig. 2. (a) show a significant increase in transmission $\operatorname{Tr} \sim$ $2 \%(\sim 43.9 \mathrm{~dB} / \mathrm{cm})$ to $\operatorname{Tr} \sim 43 \%(\sim 9.5 \mathrm{~dB} / \mathrm{cm})$ at $\lambda=900 \mathrm{~nm}$ after annealing. The results show increasing transmission in the near infrared (NIR). A Fourier Transform Infrared (FTIR) spectrometer was used to investigate transmission properties further into the NIR, Near IR-FTIR is a useful technique for the rapid and accurate identification of polymer materials [21]. Fig. 2. (b) shows the spectra for the annealed and non-annealed samples.
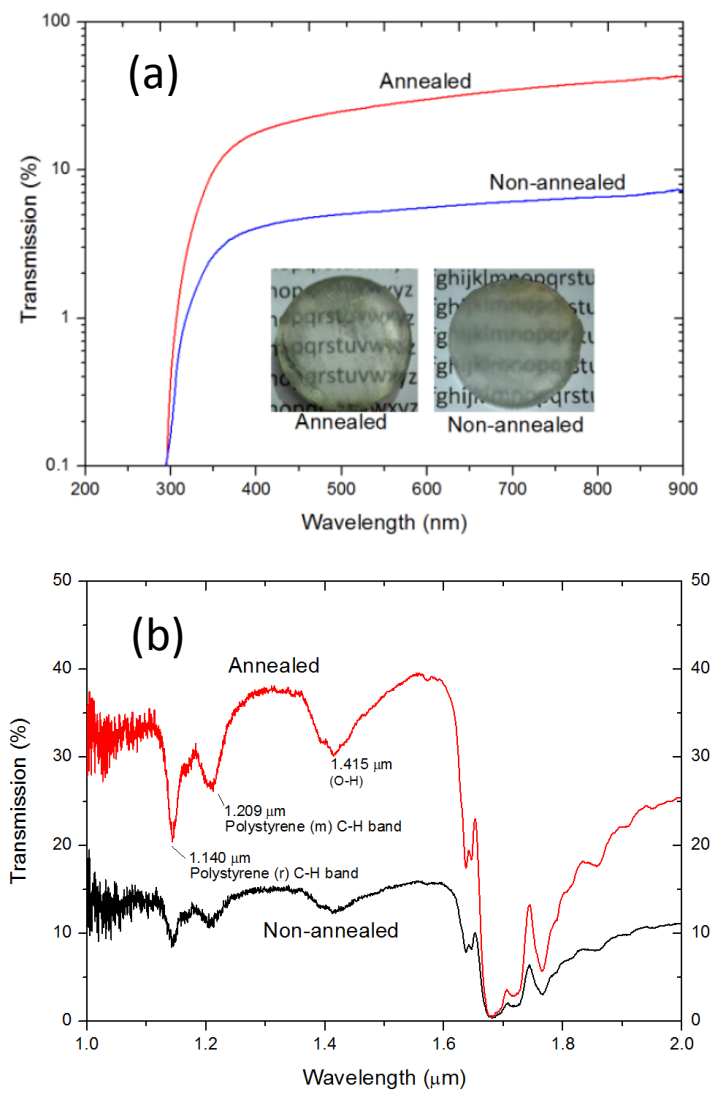

Fig. 2. Transmission (Tr) spectra and photos of the annealed and non-annealed preform: (a) UV-Visible spectra taken with a spectrophotometer; (b) IR spectra taken using a FTIR spectrometer indicating 2 polystyrene overtones for the aryl (r) and methelnic (m) $\mathrm{C}-\mathrm{H}$ bands as well as a characteristic $\mathrm{O}-\mathrm{H}$ band.

The characteristic C-H overtone bands of polystyrene are clearlydiscernable at $\lambda=1.140 \mu \mathrm{m}$ and $1.209 \mu \mathrm{m}$, as is significant $0-\mathrm{H}$ 
absorption in the region $\lambda=1.415 \mu \mathrm{m}$. Most notable, however, is the increase in transmission over the spectral window of $\Delta \lambda=(1500-$ 1600) $\mathrm{nm}$; this is an important transmission band for telecommunications. Above this region the transmission is observed to fall-off rapidly, a common feature exhibited by other polymers such as acrylic and polycarbonate-based materials [22]. The air-structured preform was drawn into fibre using a draw tower with a lowtemperature radiative furnace. The preform is passed through the furnace at a feed speed $v=1 \mathrm{~mm} / \mathrm{min}$. No gases were required to pressurise the holes and the temperature of the furnace was stabilized at $T=160{ }^{\circ} \mathrm{C}$. The preform was pulled out of the furnace into fibre at a rate $v=35 \mathrm{~mm} / \mathrm{min}$. Once the process is optimised, it was possible to spool a fibre length of up to $L \sim 10 \mathrm{~m}$. The preform experienced a small degree of bulging (so-called "mushrooming") inside the furnace during the first few minutes of drawing. This deformation led to the preform becoming slightly elliptical in shape. The likely causes involve incomplete polymerisation during printing as well as trapped air and moisture.

Optical microscope images of the final fibre cross section are shown Fig. 3. (a) and (b). A length of fibre $L=15 \mathrm{~cm}$ is cleaved and then illuminated from one end by a broadband white light source. The other end of the fibre is then imaged using red and green bandpass filters. A number of air bubbles are clearly discernable in these images, but improvements on printer settings such as resolution and printing speeds, as well as additional preform pre-annealing, can help to eliminate or minimise these defects. The outer diameter of the fibre is measured to be $\phi_{O D 1} \sim 712 \mu \mathrm{m}$ and $\phi_{O D 2} \sim 605 \mu \mathrm{m}$ for the major and minor axes respectively, whilst the core diameter is $\phi_{c 1} \sim 221 \mu \mathrm{m}$ and $\phi_{c 2} \sim 148 \mu \mathrm{m}$ for the major and minor axes respectively. A photo of white light output of the fibre projected onto a screen $\sim 1 \mathrm{~cm}$ from the fibre end-face is shown in Fig. 3. (c).
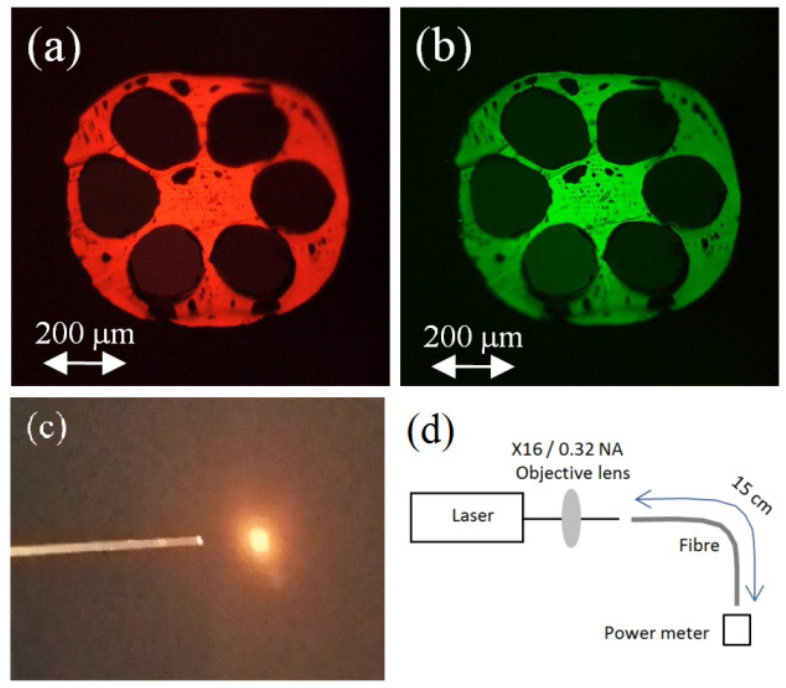

(d)

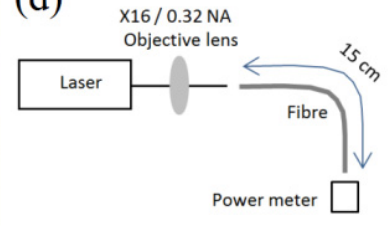

Fig. 3. Transmission of light through the drawn optical fibre: (a) 630 $\mathrm{nm}$ and (b) $515 \mathrm{~nm}$ guided light imaged at the fibre end face; (c) white light output projected onto a screen; (d) Schematic of set-up used for guidance test.

In order to assess the optical guidance properties of the fibre, the output of a HeNe laser $(\lambda=632.8 \mathrm{~nm})$, a Nd:YAG laser $(\lambda=1064 \mathrm{~nm})$ and the line of a tunable semiconductor laser $(\lambda=1550 \mathrm{~nm})$ were each, in turn, coupled into the fibre and the output power measured. $\mathrm{A} \times 16$ magnification lens with a numerical aperture $N A=0.32$ was used to launch light into the fibre as shown in Fig. 3. (d). The fibre was fixed in place so as to make a $90^{\circ}$ turn with a radius of curvature $r \sim 6 \mathrm{~cm}$. A schematic of the set-up is shown in Fig. 3.(d). This simple experiment reveals upper estimates of the propagation loss, $\alpha$, at each wavelength: $\alpha \sim 1.5 \mathrm{~dB} / \mathrm{cm} @ 632 \mathrm{~nm}, \alpha \sim 0.75 \mathrm{~dB} / \mathrm{cm} @ 1064 \mathrm{~nm}$, and $\alpha \sim 1.51$
$\mathrm{dB} / \mathrm{cm} @ 1550 \mathrm{~nm}$. (More accurate measurements using the cut-back method on fibre lengths of several meters will be performed when losses are decreased). These loss values compare favorably to structured optical fibres produced by capillary stacking and drilling, particularly given the large interstitial spacing between holes [23] However, due to the large cross-sectional area and large strand thickness between holes (as thick as $23 \mu \mathrm{m}$ in some places) the fibre is highly multimode and the light is not confined to the core. Increased confinement losses are therefore caused when the outer surface of the fibre comes into contact with other material. Ideally, confinement losses estimated for a single ring of holes in a lossless material are on the order of $\alpha \sim 0.2 \mathrm{~dB} / \mathrm{cm}$ for the fundamental mode and $\alpha \sim 2 \times 10^{4}$ $\mathrm{dB} / \mathrm{cm}$ for the first higher order mode [24], so significant scope for improvement remains. Improvements in annealing and also drawing parameters should allow the realisation of a thinner fibre with less scattering losses and the confinement of a single mode or multiple modes to the core.

In conclusion the first design and fabrication of an optical fibre using a preform made with 3D printing technology has been demonstrated. The approach is simple and harnesses the power of a 3D printer to print shapes of arbitrary geometries, avoiding the problems often associated with traditional preform fabrication. Although the printing time was several hours and the printing resolution low using a lowcost thermal based printer, it was automated and there were no additional steps required by an operator, achieving a huge saving in person-hours. With minimal preparation time required beforehand using user-friendly CAD software (Inventor), a fabricator would only need 30 minutes or so to design and start printing an air-structured preform depending on the complexity of the design (when printing is started, no further human involvement is required). The ability to fabricate custom 3D printing filaments made possible by extruder technology is also another exciting possibility and gives scope to easily introduce dopants into fibres; the use of multi extruder tips will allow multiple materials to be combined within the one preform, including multiple cores and much more. Of more immediate benefit to plastic research for biosensing, terahertz, metamaterials and so on, we have also shown that the modified butadiene plastic is suitable for polymer optical fibre work.

This work has focused on using low cost thermal fused deposition of polymeric material serving as a demonstration of the technology potential. The low cost of the printer means it has, at (50-100) $\mu \mathrm{m}$, too low a resolution to fabricate channels smaller than that described here. Higher quality printers, especially optical based systems, can address this problem, improving resolution and allowing multiple rings to be fabricated and much smaller hole-to-hole spacings. New approaches such as CLIP will also speed processes as well as improve complexity [5]. Whist the printer used in this paper is limited to printing polymer materials, laser based sintering and other developing methods will allow materials such as silica to come on line. We have shown that 3D printing technology is clearly a disruptive technology set to revolutionize optical fibre manufacture.

Funding. Australian Research Council (ARC) (FT110100116, DP140100975, LE0883038, LE100100098).

Acknowledgments. Zane Reid acknowledges an iPL summer scholarship; Md Arafat Hossain acknowledges an Australian Postgraduate Award. This work was performed in part at the Optofab node of the Australian National Fabrication Facility (ANFF) using Commonwealth and NSW State Government funding. 


\section{References}

1. H. Lipson, M. Kurman, "Fabricated: The new world of 3D printing," John Wiley \& Sons, (2013).

2.S.S. Crump, "Apparatus and method for creating three-dimensional objects," US Patent 5121329 A, (1992).

3. Md. A. Hossain, J. Canning, S. Ast, K. Cook, P. J. Rutledge and A. Jamalipour, "Combined "dual" absorption and fluorescence smartphone spectrometers", Opt. Lett., 40(8), pp 1737-1740, (2015).

4. E. D. Dickens, Jr., B. L. Lee, G. A. Taylor, A. J. Magistro, H. Ng, "Sinterable semi-crystalline powder and near-fully dense article formed therewith," US Patent 5990268 A, (1999).

5. www.carbon3d.com

6. K. Doyle, "Bioprinting: From Patches to Parts," Genetic Engineering \& Biotechnology News. 34(10), 1, pp 34-352014, (2014).

7. www.luxexcel.com

8. T. Pereira, S. Rusinkiewicz, W. Matusik, "Computational light routing: 3D printed optical fibers for sensing and display," ACM Transactions on Graphics (TOG) TOG Homepage archive, 33, 3, Article No. 24, (2014).

9. K. Willis, E. Brockmeyer, S. Hudson, I. Poupyrev, "Printed optics: 3D printing of embedded optical elements for interactive devices," UIST '12 Proceedings of the 25th annual ACM symposium on User interface software and technology, pp 589-598, (2012).

10. http://www.3ders.org/articles/20150622-micron-3dp-announcesbreakthrough-in-3d-printing-glass-materials.html

11. http://3dprinting.com/news/hp-working-3d-glass-printer/

12. J. C. Knight, T. A. Birks, P. St. J. Russell, and D. M. Atkin, "All-silica singlemode optical fiber with photonic crystal cladding," Opt. Lett. 21, pp 15471549, (1996).

13. J. Canning, E. Buckley and K. Lyytikainen, "Propagation in air by field superposition of scattered light within a Fresnel fibre," Opt. Lett. 28(4), pp 230-232, (2003).

14. C. Martelli, J. Canning, "Fresnel fibres with omni-directional zone crosssections," Opt. Exp., 15(7), pp 4281-4286, (2007).

15. S. Huntington, B. Gibson, J. Canning, K. Digweed-Lyytikainen, J. Love, V. Steblina, "A fractal based fibre for ultra-high throughput optical probes," Opt. Express, 15(5), pp 2468-2475, (2007).

16. K. M. Kiang, K. Frampton, T. M. Monro, R. Moore, J. Tucknott, D. W. Hewak, D. J. Richardson, H. N. Rutt, “Extruded single mode non-silica glass holey optical fibres," Electron. Lett., 38, pp 546-547, (2002).

17. I. A. Maxwell, "Method of optical fibre preform manufacture," US Patent US20050036731 A1, (2005).

18. P. Hambley, J. Canning, "Preliminary evaluation of graded index microstructured polymer optical fibre (GIMPOF) for LAN applications," Australian Conf. on Optical Fibre Technology (ACOFT 2007), Melbourne, Australia, (2007)

19. M. S. Milczeswki, H. J. Kalinowski, J. C. C. Silva, J. A. Simões, J. Canning, C. Martelli, "Determination of orthodontics forces using optic fibre sensors," 83rd Congress of European Orthodontics Society (EOS 2007), Berlin Germany, (2007).

20. Y. Li and H. Shimizu, "Improvement in toughness of poly(l-lactide) (PLLA) through reactive blending with acrylonitrile-butadiene-styrene copolymer (ABS): Morphology and properties," Eur. Polym. J., 45, pp 738746, (2009).

21. N. Eisenreich, H. Kull, E. Thinnes, "Fast identification of plastic materials by near-infrared spectroscopy," Krause, H.H.; Penninger, J.M.L., Conversion of Polymer Wastes and Energetics, Toronto-Scarborough: ChemTec Publishing, pp 21-30, (1994).

22. M. J. Weber, "Handbook of optical materials," CRC Press, pp 296-297, (2003).

23. A. Argyros, "Microstructured polymer optical fibers," J. Lightwave Technol. 27, pp 1571-1579, (2009).

24. A. P. White, R. C. McPhedran, C. M. de Sterke, L. C. Botten and M. J. Steel, "Confinement losses in microstructured optical fibres," Opt. Lett. 26, pp 1660-1662, (2001). 